Article

\title{
Immediate Effect of Whole-Body Vibration on Skin Temperature and Lower-Limb Blood Flow in Older Adults with Type 2 Diabetes: Pilot Study
}

\author{
Kennedy Freitas Pereira Alves ${ }^{1}$ (D), Ana Paula de Lima Ferreira ${ }^{1}$, \\ Luana Caroline de Oliveira Parente ${ }^{1}$, François Talles Medeiros Rodrigues ${ }^{1}$, \\ Thais Vitorino Marques ${ }^{1}$, Gabriel Barreto Antonino ${ }^{1}$, Luiz Carlos de Melo ${ }^{1}$, \\ Débora Wanderley Villela ${ }^{1}$, Marcelo Renato Guerino ${ }^{1}$, Wagner Souza Leite ${ }^{1}$, \\ Shirley Lima Campos ${ }^{1}$, Larissa Coutinho de Lucena ${ }^{2}, \operatorname{Redha} \operatorname{Taiar}^{3} \mathbb{D}$ and \\ Maria das Graças Rodrigues de Araújo 1,*(D)
}

1 Department of Physical Therapy, Universidade Federal de Pernambuco (UFPE), Recife-PE 50670-901, Brazil; kennedyfpa@hotmail.com (K.F.P.A.); apllima@yahoo.com.br (A.P.d.L.F.); luanaparente00@gmail.com (L.C.d.O.P.); francoismedeirosfisiot@gmail.com (F.T.M.R.); thaisvmarques@hotmail.com (T.V.M.); gabrielbarreto@live.com (G.B.A.); luiz-carlos-melo@hotmail.com (L.C.d.M.); deborawanderley84@hotmail.com (D.W.V.); marceloguerino@hotmail.com (M.R.G.); wagnerszleite@gmail.com (W.S.L.); shirleylcampos@uol.com.br (S.L.C.)

2 Faculdade Nova Esperança (FACENE), João Pessoa-PB 58067-698, Brazil; larissacoutinho@gmail.com

3 GRESPI, Université de Reims Champagne-Ardenne, 51100 Reims, France; rtaiar@chu-reims.fr

* Correspondence: mgra@ufpe.br

Received: 20 December 2019; Accepted: 11 January 2020; Published: 19 January 2020

\begin{abstract}
The purpose of this study was to evaluate the response of a single whole-body vibration (WBV) training session to peripheral skin temperature and peripheral blood flow of older adults with type 2 diabetes. A double-blind, controlled clinical trial was conducted following the Consolidated Standards of Reporting Trials (CONSORT) guidelines. A single session of WBV (24 Hz; amplitude $4 \mathrm{~mm}$; vibration time $45 \mathrm{~s}$, with a series of eight repetitions with recovery between repetitions of $30 \mathrm{~s}$; total time of $10 \mathrm{~min}$ ) or sham vibration on the Kikos P204 Vibrating Platform was employed. To assess skin temperature, the FLIR E40bxs thermographic camera and the ultrasonic vascular Doppler for flow velocity were used. Evaluation occurred before and after a WBV or sham intervention. The sample consisted of three men and 17 women. In the WBV group, there was a decrease in the temperature from $29.7^{\circ} \mathrm{C}( \pm 1.83)$ to $26.6^{\circ} \mathrm{C}( \pm 2.27)$, with $p=0.01$. Temperature following sham decreased from $28.6{ }^{\circ} \mathrm{C}( \pm 1.84)$ to $26.3^{\circ} \mathrm{C}( \pm 2.49)$, with $p=0.01$. Regarding blood flow, there was a decrease in the analyzed arteries, especially the left posterior tibial artery, where there was a statistically significant flow reduction from $27.1 \mathrm{~m} / \mathrm{s}( \pm 25.36)$ to $20.5 \mathrm{~m} / \mathrm{s}( \pm 19.66)$, post WBV $(p=0.01)$. In the sham group, an increased flow velocity was observed for all the arteries analyzed, except for the left dorsal artery. Immediately following a full-body vibration session, peripheral skin temperature and lower-limb blood flow tend to decrease in diabetic patients. However, from the design of study developed, we cannot infer the maintenance of this effect in the medium and long term.
\end{abstract}

Keywords: type 2 diabetes mellitus; blood flow velocity; skin temperature; vibration

\section{Introduction}

Diabetes mellitus (DM) is one of the largest global health emergencies of the 21st century and, each year, more people develop and live with this condition. In addition to the 415 million adults 
diagnosed with diabetes, there are 318 million adults with glucose intolerance, resulting in a high risk of aggravating the condition and improving future disease development [1]. In Brazil, the number increased from 4.5 million in 2000 to 11.3 million in 2013, with an estimated range of 19.2 million by 2035 [2].

DM is a metabolic disorder of multiple etiologies characterized by hyperglycemia, which may be caused by defects in insulin secretion and/or action, involving specific pathological causes, such as the destruction of pancreatic $\beta$-cells responsible for the production of this hormone. This condition generates numerous side effects, such as the consequent disturbance mainly of carbohydrates, as well as proteins and fats, which leads to complications in various organs and may be accompanied by dyslipidemia, systemic arterial hypertension ( $\mathrm{SAH})$, endothelial dysfunction, obesity, smoking, and physical inactivity. Other macrovascular complications are either myocardial infarction and stroke, whereas microvascular complications include retinopathies and nephropathies [3-6].

The classification of the Brazilian Diabetes Society (BDS) includes four types defined according to specific disabilities or processes. Type $2 \mathrm{DM}$ has an unknown etiology and is linked to relative insulin deficiency when it has a state of resistance combined with failure to secrete insulin, requiring the use of synthetic insulin to achieve glycemic control. It covers about $90 \%$ of diabetes cases in the population and usually manifests in adults aged over 45 with overweight or central fat deposition, physical inactivity, and family history of type 2DM [6,7].

Endothelial dysfunction is an important early factor in the development of macrovascular and microvascular complications of diabetes. Vascular endothelium is a dynamic tissue lining the blood vessels and the heart, and its performance is essential for homeostasis. The endothelium has several functions like maintenance of vascular tone, intravascular homeostasis, participation in the inflammatory process, and maintenance of blood flow. Reduction in peripheral blood flow and, consequently, local cooling of the skin are relevant markers of endothelial dysfunction that can be used as predecessors of cardiovascular disease. In older adults with type 2 diabetes, vascularization of the lower limbs is compromised [7-9]. The basal skin temperature in patients with type 2 diabetes is significantly lower than in patients without diabetes [10]. and this fact can be explained by the dilation of arteriovenous anastomoses and reduced blood supply in the capillaries due to autonomic neuropathy [11].

Many studies showed reduced blood glucose, insulin resistance, dyslipidemia, hypertension, and improved vascular function after regular physical activity, which are recognized as an important tool in diabetes control. Evidence of vasodilation after aerobic exercise and resistance training in diabetic individuals suggests increased vascular diameter and glucose uptake in the musculoskeletal system, which contributes to blood supply and lower-limb irrigation [12-15].

Reduced exercise tolerance is an important feature in patient with type 2 diabetes, who often have muscle weakness, making exercise more challenging. Whole-body vibration (WBV) is an alternative therapy which consists of a training program that exposes the whole body to mechanical vibrations. It was shown to be a safe alternative intervention to stimulate balance, and to improve muscle strength, body composition, and endurance in sedentary individuals who cannot perform conventional exercises such as walking and functional training. Protocols vary according to number of sessions per week, number of weeks, and frequency used in WBV in hertz (Hz) and amplitude (mm) [16-19].

However, there are few reports in the literature about the immediate effect of WBV on skin temperature and blood flow in the lower limbs of older adults with type 2 diabetes. Thus, the aim of this study was to evaluate the response of a single whole-body vibration training session to peripheral blood circulation and peripheral skin temperature in older adults with type 2 diabetes.

\section{Methods}

This is a pilot, randomized, double-blind, controlled clinical study that followed the guidelines set out in the Consolidated Standards of Reporting Trials (CONSORT) conducted with patients diagnosed with type 2 diabetes mellitus. 
The research was carried out in the Laboratório de Cinesioterapia e Recursos Terapêuticos Manuais (LACIRTEM) of the Physiotherapy Department of the Universidade Federal de Pernambuco (UFPE). This study was approved by the Research Ethics Committee of the CCS/UFPE (No.3.219.332) and registered at www.clinicaltrials.gov (NCT04207853), respecting the ethical aspects of Resolution 466/12 of the National Health Council and the Declaration of Helsinki. All participants signed the free and informed consent form (FICF) after being informed of the objectives, risks, and benefits of the research.

The study was disseminated in various ways to attract volunteers (social networks, leaflets, lectures, scientific events, etc.). Volunteers were instructed to contact the research team to receive information and study guidance. When the volunteer met the research criteria, they were instructed to attend the day and time scheduled for exams to confirm the disease, while wearing appropriate clothes for the study.

The study included 20 clinically classified type 2 diabetic men (three) and women (17) who were aged 60 to 80 years, functionally independent, without severe foot deformities requiring the use of therapeutic footwear, without orthopedic deficiencies, without indication of deep vein thrombosis, without the use of walking aids, and with cognitive ability to respond and perform the exercises assessed by the Mini-Mental State Examination (MMSE), using 19 points as the cutoff point for illiteracy [20].

Volunteers who performed another physical activity and those who presented peaks of hypertension during treatment or who reported discomfort during the session that prevented the completion of training were discontinued.

Randomization was performed by a researcher not involved in any research stage, using the tool offered by www.randomization.com, and the order of participants was placed in numbered, sealed, and opaque envelopes, which were only opened at the time of the survey intervention. Thus, participants were randomly divided into two groups: whole-body vibration training (WBV), simulated vibration training (sham), and a single treatment session for WBV and sham. All participants underwent an initial and final evaluation.

Initially, anthropometric data were collected: gender, age, body mass index (BMI), vital signs (heart rate (HR), blood pressure (BP), and peripheral oxygen saturation $\left(\mathrm{SpO}_{2}\right)$, capillary glucose, and clinical history at the time of evaluation. Subsequently, the volunteers were assessed for peripheral circulation through blood flow and peripheral skin temperature on both limbs and then instructed to perform training on the vibrating platform. At the end of the intervention, blood flow, peripheral skin temperature, vital signs, and capillary blood glucose were re-evaluated.

\subsection{Vital Signs}

Heart rate (HR) measured in beats per minute (BPM) and peripheral oxygen saturation $\left(\mathrm{SpO}_{2}\right)$ expressed as a percentage (\%) were evaluated using a Contec model CMS50M digital pulse oximeter (Contec Medical System Co., Ltd., Qinhuangdao, Hebei Province, China) placed on the second left toe with pressure. Blood pressure (BP) was measured in millimeters of mercury ( $\mathrm{mmHg}$ ) using a PREMIUM aneroid sphygmomanometer and a Rappaport Premium adult stethoscope (Accumed-Produtos Medico-Hospitalares Ltda, Rio de Janeiro-RJ, Brazil) using the left arm supported during measurement, with the patient sitting comfortably after $15 \mathrm{~min}$ of rest at baseline and immediately after the intervention.

\subsection{Capillary Blood Glucose}

Capillary blood glucose expressed in milligrams per deciliter ( $\mathrm{mg} / \mathrm{dL}$ ) was verified by an Accu-Chek Active GU blood glucose monitor (Roche Diabetes Care Ltda, São Paulo-SP, Brazil), with the participant sitting comfortably with their arms resting on their legs before and at the end of each intervention to monitor and control the patient's glycemic index. 


\subsection{Anthropometric Data}

Body composition was evaluated using a digital electronic anthropometric scale with a retractable ruler model MIC 200PPA (Micheletti Industria e Comercio de Equipamentos EIRELI, São Paulo-SP, Brazil). Body mass index (BMI) was defined as weight in kilograms divided by the square of height in meters $\left(\mathrm{kg} / \mathrm{m}^{2}\right)$.

\subsection{Blood Flow Velocity}

The evaluation of blood flow velocity expressed in meters per second $(\mathrm{m} / \mathrm{s})$ was performed by ultrasonic vascular Doppler BV-620VP (Shenzhen Bestman Instarument Co., Ltd., Shenzhen, Guangdong, China), with sine wave emission. Prior to evaluation, a wide trichotomy of the examination site was performed, and then the area was coated with a water-soluble gel to promote better contact between the transducer and the skin. The transducer was positioned at a $45^{\circ}$ angle to the blood vessels and was analyzed for flow. For the posterior tibial artery and the dorsal artery of the foot, recordings of the high-frequency transducer $(8 \mathrm{~Hz})$ were made, using the distal third of the calf and the instep as a reference. Confirmation of the most appropriate site for the evaluation of each blood vessel was performed through the acoustic signals generated by the equipment and, at this point, a marking was made to facilitate re-evaluation after the intervention. Three measurements were taken at 30-s intervals, and the final result was the average of the values obtained in the three collections. The exam was repeated after the intervention via which the patient was submitted, respecting the markings of the previous collection.

\subsection{Skin Temperature}

Skin temperature was evaluated using an FLIR E40bx thermographic camera (FLIR Systems Inc., Wilsonville, OR, USA) with a thermal sensitivity of $0.05^{\circ} \mathrm{C}$ and a resolution of $160 \times 120$ pixels. The camera was positioned at a distance of $1 \mathrm{~m}$ from the assessed region, at an angle of $90^{\circ}$ to the camera lens. Participants were instructed regardingthe preparatory measures for the thermographic exam, according to the recommendations of Priego Quesada (2017). Prior to the exam, the volunteers rested for $10 \mathrm{~min}$ in a room with a temperature between 18 and $23{ }^{\circ} \mathrm{C}$ and relative humidity between $40 \%$ and $70 \%$, monitored with the help of athermo-hygrometer (KT-908). Reflected temperature was measured by the "reflective method" described in ISO 18434-1: 2008 for thermographic camera calibration [21]. We noted that the human body emissivity is assumed to be constant (about 0.98) [22] to ensure that the influence of the environment reflection is negligible.

Image analyses were performed according to the Glamorgan (2008) protocol for the anterior and posterior leg and dorsal region and sole of the feet, using the average temperature of a polygon, which followed the contour of the studied areas [23].

Images were collected before and after the intervention. Subsequently, the images obtained were organized, stored, and analyzed in the FLIR Tools software (FLIR Systems Inc., Wilsonville, OR, USA) provided by the camera manufacturer (Figure 1).

\subsection{Whole-Body Vibration}

The vibratory platform used was the Kikos P204-110v (Kikos Fitness, São Paulo-SP, Brazil), whose vibration direction is of the lateral oscillatory type. The vibrating plate features non-slip material on its surface for better foot attachment at the time of training. The established vibration frequency was $24 \mathrm{~Hz}$. The 4-mm displacement peak was maintained throughout the program and was determined by the width of the volunteers' position on the platform. The intervention time was $45 \mathrm{~s}$, with eight series being performed and, for each, the recovery time was maintained at $30 \mathrm{~s}$ for all intervals, totaling $600 \mathrm{~s}$ of intervention. 
The subjects, barefoot, adopted a $40^{\circ}$ squat position, verified with a universal goniometer. The calcans were supported on a silicone insole, consisting of a particular type of semi-rigid sole used to correct the distribution of body weight and biomechanical changes found in the foot. Only the forefoot and midfoot were in direct contact with the platform in order to reduce the transmission of vibrations to the head. This posture was used for the both groups.

The simulated vibration "sham" was performed with the platform disconnected. A sound device was connected producing a noise similar to the connected platform for a time equivalent to the treatment protocol because the vibratory stimulus could not be visibly distinguished. Participants who experienced false vibration had no contact with those who performed the protocol effectively.

On the day of intervention, participants were instructed not to do intense exercises, requiring at least one week apart from the last moderate/high-intensity physical activity. In addition, all participants were instructed to wear comfortable and appropriate exercise clothing.

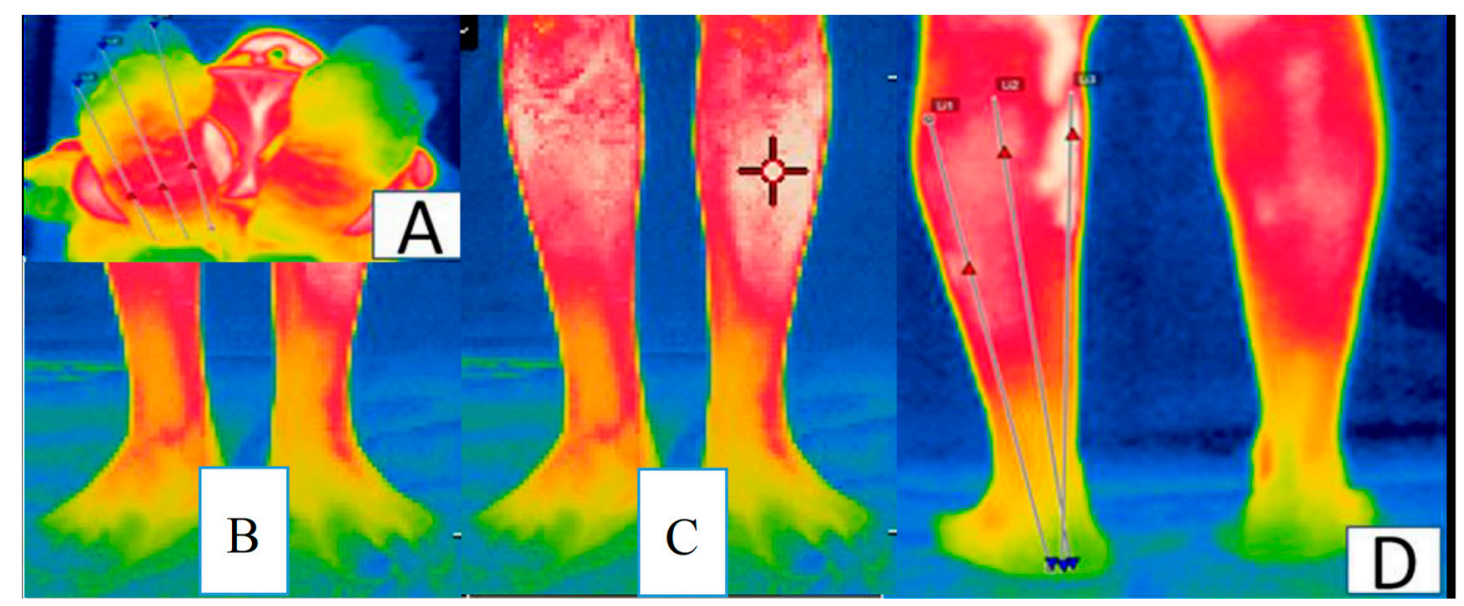

Figure 1. Regions of interest for infrared thermographic imaging: (A) sole of the feet; (B) back of the feet; (C) anterior leg; (D) back of the legs.

\subsection{Data Analysis}

Statistical analysis was performed using the statistical software SPSS version 23.0 (IMB Software Group, Chicago, IL, USA). To test the hypothesis of normality of each quantitative variable, the Shapiro-Wilk adherence test was used, and then the behavior of the quantitative variables was evaluated using the Mann-Whitney and Wilcoxon comparison test for the inter- and intragroup analysis, respectively.

The effect size was calculated by Cohen's $d$. An effect size of 0.2 was considered a small effect, an effect size of 0.5 was considered a moderate effect, and an effect size of 0.8 was considered a large effect. Descriptive statistical analysis was performed using the mean, standard deviation (SD), and percentage for qualitative variables. All tests were assigned a significance level of $95 \%(p<0.05)$.

\section{Results}

Each group consisted of 10 participants, from WBV (two men and eight women) and sham (one man and nine women), as shown in Figure 2. For all variables, the groups were considered statistically comparable at baseline, as shown in Table 1). 


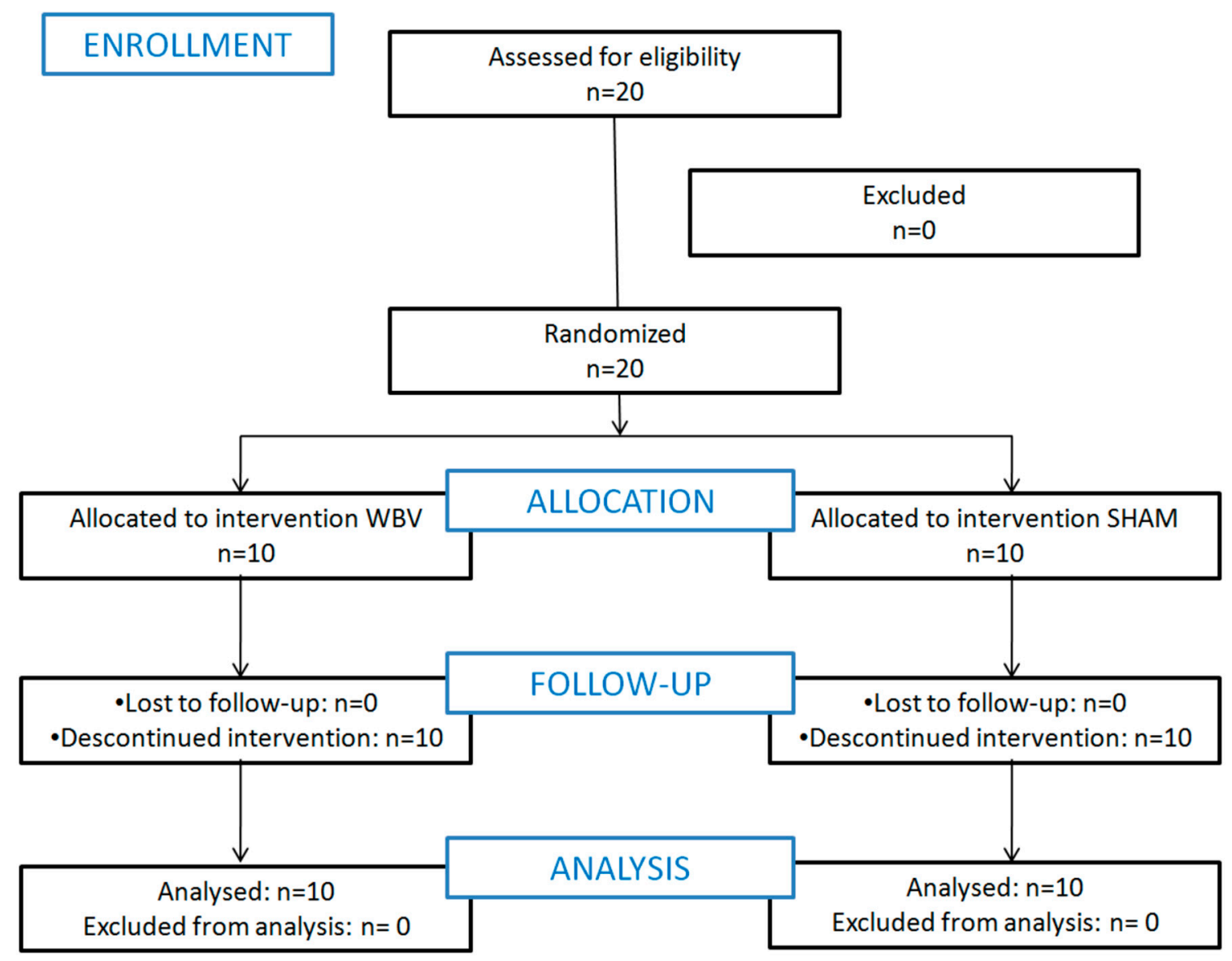

Figure 2. Flow chart which describes the recruitment, randomization, and allocation of patients.

Table 1. Baseline characteristics of patients in the whole-body vibration (WBV) and sham groups.

\begin{tabular}{cccc}
\hline & WBV & Sham & \\
\cline { 2 - 3 } Variables & $\begin{array}{c}\text { G1 } \\
(\mathbf{n = 1 0 )}\end{array}$ & $\begin{array}{c}\text { G2 } \\
(\mathbf{n = 1 0 )}\end{array}$ & \\
& $64.4( \pm 3.99)$ & $65.2( \pm 4.54)$ & 0.83 \\
\hline Age (years) & $7.29( \pm 4.53)$ & $9.13( \pm 6.81)$ & 0.55 \\
\hline Diagnostic Time (years) & $10(100 \%)$ & $10(100 \%)$ & \\
\hline Sex & $2(20 \%)$ & $1(10 \%)$ & 0.54 \\
Male & $8(80 \%)$ & $9(90 \%)$ & \\
Female & $3(30 \%)$ & $1(10 \%)$ & 0.4 \\
Smoker & $7(70 \%)$ & $9(90 \%)$ & \\
Yes & $2(28.6 \%)$ & $3(33.3 \%)$ & \\
No & - & - & \\
Former smoker & & & \\
Passive smoker & $30.4( \pm 4)$ & $27.9( \pm 3.9)$ & \\
\hline Anthropometric data & & & \\
BMI & & & \\
\hline
\end{tabular}


Table 1. Cont.

\begin{tabular}{|c|c|c|c|}
\hline \multirow[b]{2}{*}{ Variables } & WBV & Sham & \multirow[b]{2}{*}{$p$-Value } \\
\hline & $\begin{array}{c}\mathrm{G1} \\
(\mathrm{n}=10)\end{array}$ & $\begin{array}{c}\mathrm{G} 2 \\
(\mathrm{n}=10)\end{array}$ & \\
\hline \multicolumn{4}{|l|}{ Schooling } \\
\hline Illiterate & - & - & \multirow{8}{*}{0.42} \\
\hline Incomplete elementary & $1(10 \%)$ & $1(10 \%)$ & \\
\hline Complete elementary & $2(20 \%)$ & $2(20 \%)$ & \\
\hline Incomplete medium & - & $1(10 \%)$ & \\
\hline Medium complete & $2(20 \%)$ & $4(40 \%)$ & \\
\hline Incomplete higher & $2(20 \%)$ & $1(10 \%)$ & \\
\hline Graduated & $3(30 \%)$ & - & \\
\hline Postgraduate & - & $1(10 \%)$ & \\
\hline \multicolumn{3}{|l|}{ Marital status } & \multirow{5}{*}{0.24} \\
\hline Single & $3(30 \%)$ & - & \\
\hline Married & $4(40 \%)$ & $4(40 \%)$ & \\
\hline Divorced & $2(20 \%)$ & $3(30 \%)$ & \\
\hline Widowed & $1(10 \%)$ & $3(30 \%)$ & \\
\hline \multicolumn{3}{|l|}{ Family income } & \multirow{5}{*}{1} \\
\hline$<1 \mathrm{MW}$ & $3(30 \%)$ & $3(30 \%)$ & \\
\hline 1-3 MW & $4(40 \%)$ & $4(40 \%)$ & \\
\hline 4-6 MW & $2(20 \%)$ & $2(20 \%)$ & \\
\hline$>7 \mathrm{MW}$ & $1(10 \%)$ & $1(10 \%)$ & \\
\hline HPAQ & $8.49( \pm 1.19)$ & $7.9( \pm 1.37)$ & 0.32 \\
\hline \multicolumn{3}{|l|}{ Blood pressure } & \\
\hline SBP & $120( \pm 9.43)$ & $116( \pm 6.99)$ & 0.3 \\
\hline DBP & $77( \pm 8.23)$ & $78( \pm 9.66)$ & 0.81 \\
\hline Capillary blood glucose & $133.6( \pm 36.75)$ & $135.4( \pm 35.2)$ & 0.91 \\
\hline $\mathrm{SpO}_{2}$ & $98.3( \pm 0.82)$ & $95.3(4.8)$ & 0.08 \\
\hline Diabetes knowledge & $20.85( \pm 11.27)$ & $21.42( \pm 9.54)$ & 0.9 \\
\hline
\end{tabular}

Data are expressed as mean ( \pm standard deviation) or absolute frequency (relative frequency). Abbreviations: body mass index, $\mathrm{BMI}$; minimum wage, $\mathrm{MW}$; $\mathrm{SpO}_{2}$, peripheral oxygen saturation; $\mathrm{QAFH}$, usual physical activity questionnaire; BMI, body mass index; SBP, systolic blood pressure; DBP, diastolic blood pressure.

Only the plantar region of the right foot suffered a statistically significant reduction in skin temperature in both groups. In the WBV group, there was a reduction from $29.7^{\circ} \mathrm{C}( \pm 1.83)$ to $26.6^{\circ} \mathrm{C}$ $( \pm 2.27), p=0.01$. In the sham group, the reduction was from $28.6^{\circ} \mathrm{C}( \pm 1.84)$ to $26.3^{\circ} \mathrm{C}( \pm 2.49), p=0.01$. There was no statistically significant difference between the groups. For all results, the effect size was considered low. Thermographic data are expressed in Table 2.

Table 2. Mean skin temperature analysis in ${ }^{\circ} \mathrm{C}$ (and standard deviation) before and after intervention (intra- and intergroup) and effect size.

\begin{tabular}{|c|c|c|c|c|c|c|c|c|}
\hline \multirow{2}{*}{ Variables } & \multicolumn{3}{|c|}{ WBV } & \multicolumn{3}{|c|}{ Sham } & \multirow{2}{*}{ Effect Size } & \multirow{2}{*}{$p$-Value } \\
\hline & Before & Post & $p^{+}$ & Before & Post & $p^{+}$ & & \\
\hline Plant right foot & $29.7( \pm 1.83)$ & $26.6( \pm 2.27)$ & $0.01 *$ & $28.6( \pm 1.84)$ & $26.3( \pm 2.49)$ & 0.01 * & 0.12 & 0.78 \\
\hline Left foot plant & $26.9( \pm 2.33)$ & $26.7( \pm 1.89)$ & 0.84 & $28.7( \pm 2)$ & $26.3( \pm 2.21)$ & 0.05 & 0.18 & 0.67 \\
\hline Back right foot & $29.6( \pm 1.64)$ & $29( \pm 1.7)$ & 0.33 & $30.2( \pm 1.93)$ & $29.2( \pm 1.23)$ & 0.07 & -0.16 & 0.77 \\
\hline Back left foot & $27.9( \pm 1.85)$ & $29.3( \pm 1.06)$ & 0.06 & $30.2( \pm 1.93)$ & $29.7( \pm 1.25)$ & 0.30 & -0.32 & 0.45 \\
\hline Anterior right leg & $30.1( \pm 1.59)$ & $30.1( \pm 1.59)$ & 0.52 & $30.3( \pm 1.06)$ & $30.3( \pm 1.06)$ & 0.48 & -0.19 & 0.74 \\
\hline Anterior left leg & $30.1( \pm 1.52)$ & $30.1( \pm 1.52)$ & 0.21 & $30.4( \pm 1.26)$ & $30.4( \pm 1.26)$ & 0.36 & -0.24 & 0.64 \\
\hline Posterior right leg & $30.4( \pm 0.97)$ & $30.3( \pm 1.06)$ & 0.80 & $30.4( \pm .97)$ & $30.4( \pm 0.84)$ & 1 & 0.12 & 0.82 \\
\hline Posterior left leg & $30.6( \pm 0.97)$ & $30.3( \pm 1.34)$ & 0.54 & $30.5( \pm 1.08)$ & $30.6( \pm 0.84)$ & 0.76 & -0.32 & 0.56 \\
\hline
\end{tabular}

Data are expressed as mean ( \pm standard deviation). Abbreviations: WBV, whole-body vibration; sham, dummy vibration. $p^{+}$, Wilcoxon; $p$-value, Mann-Whitney; ${ }^{*}$ statistically significant result. 
There was a reduction in blood flow in the WBV group after the intervention, but there was only a statistically significant reductionin the left tibial artery, from $27.1 \mathrm{~m} / \mathrm{s}( \pm 25.36)$ to $20.5 \mathrm{~m} / \mathrm{s}( \pm 19.66)$, $p=0.01$. In the sham group, there was only a reduction in flow velocity in the left dorsal artery and an increase in all other evaluated arteries. However, none of them had a statistically significant difference, just as there was no statistically significant difference between the groups in this variable. Alow effect size was also observed for this variable. The complete analysis of blood flow velocity is detailed in Table 3.

Table 3. Analysis of mean blood flow velocity in $\mathrm{m} / \mathrm{s}$ (and standard deviation) before and after intervention (intra and intergroup) and effect size.

\begin{tabular}{ccccccccc}
\hline \multirow{2}{*}{ Variables } & \multicolumn{3}{c}{ WBV } & \multicolumn{2}{c}{ Sham } & Effect Size & \multicolumn{1}{c}{$p$-Value } \\
\cline { 2 - 7 } & Before & Post & $p^{+}$ & Before & Post & $p^{+}$ & \\
\hline Right dorsal artery & $29.3( \pm 25.79)$ & $23.4( \pm 24.61)$ & 0.31 & $27.9( \pm 29.14)$ & $29.3( \pm 25.79)$ & 0.81 & -0.23 \\
Left dorsal artery & $24.6( \pm 21.65)$ & $20.3( \pm 20.25)$ & 0.37 & $26.8( \pm 23.58)$ & $23.8( \pm 21.21)$ & 0.13 & -0.17 & 0.61 \\
Right tibial artery & $23.1( \pm 25.92)$ & $21.4( \pm 23.68)$ & 0.58 & $21.3( \pm 24.76)$ & $23.2( \pm 26)$ & 0.06 & -0.07 & 0.87 \\
Left tibial Artery & $27.1( \pm 25.36)$ & $20.5( \pm 19.66)$ & $0.01 *$ & $24( \pm 26.24)$ & $27.2( \pm 25.42)$ & 0.69 & -0.26 & 0.52 \\
\hline
\end{tabular}

Abbreviations: WBV, whole-body vibration; sham, dummy vibration. $p^{+}$Wilcoxon; $p$-value, Mann-Whitney; * statistically significant result.

\section{Discussion}

In the present study, in both groups (WBV and sham), there was a reduction in skin temperature and blood flow velocity, corroborating previous studies. Fujiwara et al. (2000) evaluated 61 diabetic participants and 16 healthy subjects, and they reported that basal skin temperature in diabetic patients was significantly lower than in healthy patients, in addition to lower temperature variation and recovery after intervention. These findings agree with the present study, where there was little variation in some of the evaluated points and, in some of them, the temperature remained constant [10]. According to Uccioli et al. (1994), this fact can be explained by the dilation of arteriovenous anastomoses and reduced blood supply in the capillaries due to autonomic neuropathy [11].

The study by Kistler et al. (1998) inferred that capillary blood flow and blood pressure are lower in autonomic diabetic neuropathy because the anatomical walls are larger, directing flow and damaging peripheral sympathetic nerves. This promotes a slow recovery of skin temperature, justifying the smallest temperature variation in the present study [24].

Manimmanakorn et al. (2017) performed a study with a vibratory platform with 36 diabetic participants, of which 17 were trained on the platform for 12 weeks and 19 participants maintained their daily activities only. Reductions in peak systolic velocity and diastolic pressure were noticed in the WBV group, but without significant difference from the control group. In the present study, although no peak systolic velocity and diastolic pressure were evaluated, we observed a reduction in which there was a reduction in blood flow velocity in the evaluated arteries. This finding can be explained by the possible vibration-induced dilation of the smaller blood vessels present in the muscles, reducing peripheral vascular resistance by inhibiting endothelin, an important vasoconstrictor [25].

Saudoudo et al. (2013) evaluated 40 diabetics, 20 of whom underwent 12 weeks of whole-body vibration training, and reported increased blood flow velocity after 12 weeks of intervention, with a significant difference compared with the control group. This divergence in the results found in relation to the present study is possibly due to the accomplishment of a training program over a long period of time, enhancing the effects of vibration and promoting long-term results, which may not be the case in the first session of the study training [8].

Bagavathiappan et al. (2010) analyzed 112 patients diagnosed with type 2 diabetes through thermographic images of the plantar region, correlating the presence of diabetic neuropathy and the cutaneous temperature of these individuals. Higher values of mean and global foot temperature were found in patients with neuropathy, in addition to a positive correlation with temperature in the hallux regions, bilaterally. This finding may be justified by neuropathic feet being warm, with palpable pulses 
and distended veins, indicating increased blood flow, although slow, and possible congestion in this region [26].

Due to the neurovascular lesions triggered by hyperglycemia, as well as the response to lesions or infections, the vascular response of these individuals is slower, making them more susceptible to ulcer development and possible long-term necrosis and amputation. Thus, due to the increase in skin temperature after the use of WBV, it is plausible to think that the use of this equipment may be beneficial for the vascular maintenance of these individuals, and thermographic monitoring is an ally in the maintenance of these patients' lesions [26]. The findings cited are discordant with the present study.

However, Moreira-Marconi et al. (2019) evaluated 19 healthy subjects and reported a reduction in skin temperature in most participants, regardless of the frequency used in $W B V(0 \mathrm{~Hz}, 30 \mathrm{~Hz}$, and $50 \mathrm{~Hz})$. This reduction can be explained by the fast and constant isometric muscle action that promotes work and increases the body's metabolic rate, increasing intramuscular temperature, modifying the thermal balance, activating the feedback-mediated heat loss mechanism. This change leads to redirection of blood flow from inactive to active areas. Then, during vibration, blood flow redistributes to the periphery, allowing heat exchange with the external environment, cooling the skin, and causing blood to flow to the lower-temperature muscle regions, thus preventing hyperthermia and maintaining homeostasis [27].

In addition, a larger blood supply is needed in the recruited musculature, requiring greater blood supply, initially diverting from adjacent peripheral areas. Under these conditions, a vasoconstrictor response induced by the mechanical action of vibration occurs, promoting a reduction in peripheral perfusion, and reducing the local skin temperature [26]. The above findings of the study corroborate the results found in the present study.

WBV training is an exercise program that does not increase excessive physical effort to the skeletal muscle system compared to conventional exercise. Mastering this training with WBV can help with choosing the most appropriate training program for older adults with type 2 diabetes.

\section{Conclusions}

Immediately following a full-body vibration session, peripheral skin temperature and lower-limb blood flow tend to be reduced in older adults with type 2 diabetes. However, from the design of study developed, we cannot infer the maintenance of this effect in the medium and long term.

\section{Study Limitations}

As this clinical trial was a pilot with a small number of participants, this study can serve as a basis for sample size estimation in future studies, taking into account the trends pointed out in the results presented. No condition in the metabolic control of diabetes was considered because it was a single-session study, given that glycated hemoglobin is the average glycaemia in the last $3-4$ months. An increase in the sample size and a higher male presence (balanced gender repartition) in a future study should be considered to infer more robust results.

Author Contributions: Conceptualization, L.C.d.O.P., K.F.P.A. and M.d.G.R.d.A.; data curation, D.W.V. and A.P.d.L.F.; formal analysis, W.S.L. and S.L.C.; funding acquisition, M.d.G.R.d.A. and M.R.G.; Investigation, F.T.M.R., G.B.A., T.V.M. and L.C.d.O.P.; methodology, L.C.d.M., L.C.d.O.P., K.F.P.A., F.T.M.R., T.V.M., L.C.L, A.P.d.L.F., D.W.V., W.S.L. and M.d.G.R.d.A.; project administration, M.R.G., L.C.d.L. and M.d.G.R.d.A.; writing-original draft, L.C.d.O.P., K.F.P.A., G.B.A. and M.d.G.R.d.A.; writing-review and editing, K.F.P.A., S.L.C., R.T. and M.d.G.R.d.A. All authors have read and agreed to the published version of the manuscript.

Funding: This research was funded by Fundação de Amparo à Ciência e Tecnologia do Estado de Pernambuco (FACEPE) through financial support APQ No. 0337-13.

Conflicts of Interest: The authors declare that there is no conflict of interests regarding the publication of this paper. 


\section{References}

1. International Diabetes Federation IDF. IDF Atlas, Seventhedition, Brussels, Belgium. 2015. Available online: www.diabetesatlas.org (accessed on 10 November 2019).

2. Cecilio, H.P.M.; Arruda, G.O.; Teston, E.F.; Santos, A.L.; Marcon, S.S. Comportamentos e comorbidades associados às complicações microvasculares do diabetes. Acta Paul. Enferm. 2015, 28. [CrossRef]

3. Brasil. Ministério da Saúde. Secretaria de Atenção à Saúde. Departamento de Atenção Básica. Estratégias Para o Cuidado da Pessoa com Doença Crônica: Diabetes Mellitus; Ministério da Saúde, Secretaria de Atenção à Saúde. Departamento de Atenção Básica: Brasília, Brazil, 2013.

4. Del Pozo-Cruz, J.; Alfonso-Rosa, R.M.; Ugia, J.L.; McVeigh, J.G.; Del Pozo-Cruz, B.; Sañudo, B. A Primary Care-Based Randomized Controlled Trial of 12-Week Whole-Body Vibration for Balance Improvement in Type 2 Diabetes Mellitus. Arch. Phys. Med. Rehabil. 2013, 94, 2112-2118. [CrossRef] [PubMed]

5. Irvine, C.; Taylor, N.F. Progressive resistance exercise improves glycaemic control in people with type 2 diabetes mellitus: A systematic review. Aust. J. Physiother. 2009, 55, 237-246. [CrossRef]

6. Sivanandam, S.; Anburajan, M.; Venkatraman, B.; Menaka, M.; Sharath, D. Medical thermography: A diagnostic approach for type 2 diabetes based on non-contact infrared thermal imaging. Endocrine 2012, 42, 343-351. [CrossRef]

7. Sociedade Brasileira de Diabetes. Diretrizes da Sociedade Brasileira de Diabetes (2017-2018). São Paulo: Editora Clannad. 2017. Available online: http://www.diabetes.org.br/profissionais/images/2017/diretrizes/ diretrizes-sbd-2017-2018.pdf (accessed on 10 November 2019).

8. Sañudo, B.; Alfonso-Rosa, R.; De Pozo-Cruz, B.; De Pozo-Cruz, J.; Galiano, D.; E Figueroa, A. O treinamento de vibração do corpo inteiro melhora o fluxo sanguíneo das pernas e a adiposidade em pacientes com diabetes mellitus tipo 2. Eur. J. Appl. Physiol. 2013, 113, 2245-2252. [CrossRef]

9. Silber, H.A.; Lima, J.A.; Bluemke, D.A.; Astor, B.C.; Gupta, S.N.; Foo, T.K.; Ouyang, P. Arterial reactivity in lower extremities is progressively reduced as cardiovascular risk factors increase: Comparison with upper extremities using magnetic resonance imaging. J. Am. Coll. Cardiol. 2007, 49, 939-945. [CrossRef]

10. Fujiwara, Y.; Inukai, T.; Aso, Y.; Takemura, Y. Thermographic measurement of skin temperature recovery time of extremities in patients with type 2 diabetes mellitus. Exp. Clin. Endocrinol. Diabetes 2000, 108, 463-469. [CrossRef]

11. Uccioli, L.; Mormile, F.; Monticone, G.; Mennuni, G.; Durola, L.; Menzinger, G.; Russo, F. Autonomic neuropathy influences great toe blood pressure. Diabetes Care 1994, 17, 284-287. [CrossRef]

12. Sato, Y.; Nagasaki, M.; Kubota, M.; Uno, T.; Nakai, N. Clinical aspects of physical exercise for diabetes/metabolic syndrome. Diabetes Res. Clin. Pract. 2007, 77 (Suppl. S1), S87-S91. [CrossRef]

13. Chudyk, A.; Petrella, R.J. Effects of exercise on cardiovascular risk factors in type 2 diabetes: A meta-analysis. Diabetes Care 2011, 34, 1228-1237. [CrossRef]

14. Okada, S.; Hiuge, A.; Makino, H.; Nagumo, A.; Takaki, H.; Konishi, H.; Goto, Y.; Yoshimasa, Y.; Miyamoto, Y. Effect of exercise intervention on endothelial function and incidence of cardiovascular disease in patients with type 2 diabetes. J. Atheroscler. Thromb. 2010, 17, 828-833. [CrossRef] [PubMed]

15. Bloor, C.M. Angiogenesis during exercise and training. Angiogenesis 2005, 8, 263-271. [CrossRef] [PubMed]

16. Behboudi, L.; Azarbayjani, M.A.; Aghaalinejad, H.; Salavati, M. Effects of aerobic exercise and whole body vibration onglycaemia control in type 2 diabetic males. Asian J. Sports Med. 2011, 2, 83-90. [CrossRef]

17. Regensteiner, J.G. Type 2 diabetes mellitus and cardiovascular exercise performance. Rev. Endocr. Metab. Disord. 2004, 5, 269-276. [CrossRef] [PubMed]

18. Egger, A.; Niederseer, D.; Diem, G.; Finkenzeller, T.; Ledl-Kurkowski, E.; Forstner, R.; Pirich, C.; Patsch, W.; Weitgasser, R.; Niebauer, J. Different types of resistance training in patients with type 2 diabetes mellitus: Effects on glycemic control, muscle mass and strength. Eur. J. Prev. Cardiol. 2013. [CrossRef] [PubMed]

19. Machado, A.; Garcia Lupez, D.; Gonzalez Gallego, J.; Garstschea, N. Whole body vibration training increases muscle strength and mass in older women: A randomize controlled trial. Scand. J. Med. Sci. Sports 2010, 20, 200-207. [CrossRef] [PubMed]

20. Lourenço, R.A.; Veras, R.P. Mini-Exame do Estado Mental: Característicaspsicométricas em idosos ambulatoriais. Revista de Saúde Pública 2006, 40, 712-719. [CrossRef] 
21. Quesada, J.I.P.; Kunzler, M.R.; Carpes, F.P. Methodological aspects of infrared thermography in human assessment. In Application of Infrared Thermography in Sports Science. Biological and Medical Physics, Biomedical Engineering; Priego Quesada, J.I., Ed.; Springer International Publishing: Cham, Switzerland, 2017; pp. $49-67$. [CrossRef]

22. Polidori, G.; Renard, Y.; Lorimier, S.; Pron, H.; Derruau, S.; Taiar, R. Medical Infrared Thermography assistance in the surgical treatment of axillary Hidradenitis Suppurativa: A case report. Int. J. Surg. Case Rep. 2017, 34, 56-59. [CrossRef]

23. Ammer, K. The Glamorgan Protocol for recording and evaluation of thermal images of the human body. Thermol. Int. 2008, 18, 125-144.

24. Kistler, A.; Mariauzouls, C.; Von-Berlepsch, K. Fingertp temperature as an indicator for sympathetic responses. Int. J. Psychophysiol. 1998, 29, 35-41. [CrossRef]

25. Manimmanakorn, N.; Manimmanakorn, A.; Phuttharak, W.; Hamlin, M.J. Effects of whole body vibration on glycemic indices and peripheral blood flow in type II diabetic patients. Malays. J. Med. Sci. 2017, 24, 55-63. [CrossRef] [PubMed]

26. Bagavathiappan, S.; Philip, J.; Jayakumar, T.; Raj, B.; Rao, P.N.; Varalakshmi, M.; Mohan, V. Correlation between plantar foot temperature and diabetic neuropathy: A case study by using an infrared thermal imaging technique. J. Diabetes Sci. Technol. 2010, 4, 1386-1392. [CrossRef] [PubMed]

27. Moreira-Marconi, E.; Moura-Fernandes, M.C.; Lopes-Souza, P.; Teixeira-Silva, Y.; Reis-Silva, A.; Marchon, R.M. Evaluation of the temperature of posterior lower limbs skin during the whole body vibration measured by infrared thermography: Cross-sectional study analysis using linear mixed effect model. PLoS ONE 2019, 14, e0212512. [CrossRef] [PubMed]

(C) 2020 by the authors. Licensee MDPI, Basel, Switzerland. This article is an open access article distributed under the terms and conditions of the Creative Commons Attribution (CC BY) license (http://creativecommons.org/licenses/by/4.0/). 